\title{
Interstitial pulmonary disorders in indium-processing workers
}

\author{
T. Chonan*, O. Taguchi* and K. Omae ${ }^{\#}$
}

ABSTRACT: The production of indium-tin oxide has increased, owing to the increased manufacture of liquid-crystal panels. It has been reported that interstitial pneumonia occurred in two indium-processing workers; therefore, the present study aimed to evaluate whether interstitial pulmonary disorders were prevalent among indium workers.

The study was carried out in $\mathbf{1 0 8}$ male workers in the indium plant where the two interstitial pneumonia patients mentioned above were employed, and included high-resolution computed tomography (HRCT) of the lungs, pulmonary function tests and analysis of serum sialylated carbohydrate antigen KL-6 and the serum indium concentration.

Significant interstitial changes were observed in 23 indium workers on HRCT and serum KL-6 was abnormally high ( $>500 \mathrm{U} \cdot \mathrm{mL}^{-1}$ ) in $\mathbf{4 0}$ workers. Workers with serum indium concentrations in the highest quartile had significantly longer exposure periods, greater HRCT changes, lower diffusing capacity of the lung for carbon monoxide and higher KL-6 levels compared with those in the lowest quartile. The serum indium concentration was positively correlated with the KL-6 level and with the degree of HRCT changes.

In conclusion, the results of the present study indicated that serum KL-6 and high-resolution computed tomography abnormalities were prevalent among indium workers and that these abnormalities increased with the indium burden, suggesting that inhaled indium could be a potential cause of occupational lung disease.

KEYWORDS: Indium-tin oxide, interstitial pneumonia, occupational lung disease, semiconductor industry, sialylated carbohydrate antigen KL-6

I ndium-tin oxide (ITO), a sintered material consisting of $90 \%$ indium oxide and $10 \%$ tin oxide, is used to make flat-panel displays such as liquid-crystal displays and plasma display panels. Its production has been increasing during the $10 \mathrm{yrs}$, particularly in east Asia. However, indium compounds, either when inhaled as an aerosol or instilled into the trachea, have been reported to exhibit pulmonary toxicity in animal experiments [1-3]. The US National Toxicology Program [4] performed indium phosphide inhalation experiments in rats and mice for 2 yrs and concluded that there was clear evidence of an increase in pulmonary inflammation and carcinogenesis.

Recently, Homma et al. [5] reported a case of severe interstitial pneumonia accompanied by bilateral pneumothorax in a 27-yr-old male whose occupation was surface grinding of ITO. This report was followed by another milder case of pulmonary fibrosis in a 30-yr-old male who worked at the same plant [6]. Therefore, the current authors conducted a comprehensive study to detect pulmonary disorders in workers employed at the indium processing plant in Japan where the two individuals were employed.

The present study included high-resolution computed tomography (HRCT) of the lungs, pulmonary function tests and measurement of the serum indium concentration. The level of serum sialylated carbohydrate antigen KL-6 was also measured. KL-6 is a high molecular weight glycoprotein mainly produced by alveolar type II cells and it has been reported that KL-6 is, like surfactant protein (SP)-A and SP-D, a sensitive marker of interstitial lung disease [7-9]. Some of the results of the present study have been reported previously in the form of an abstract [10].

\section{METHODS AND MATERIALS}

\section{Study design and subjects}

The present study was approved by the medical professional committee that oversees the health maintenance programme of the plant.

The study was carried out in October and November 2002 in a total of 108 male workers,

\section{AFFILIATIONS}

*Dept of Medicine, Nikko Memorial Hospital, Hitachi, and

\#Dept of Preventive Medicine and Public Health, School of Medicine, Keio University, Tokyo, Japan.

CORRESPONDENCE

T. Chonan

Dept of Medicine

Nikko Memorial Hospital

2-12-8 Kamine-cho

Hitachi

Ibaraki 317-0064

Japan

Fax: 81294241216

E-mail: ttchonan@mbd.ocn.ne.jp

Received:

February 102006

Accepted after revision:

September 202006

STATEMENT OF INTEREST

None declared. 
including 81 current and 27 former workers (median (range) post-exposure period $4.6 \mathrm{yrs}$ (0.3-12.4)), aged 20-60 yrs (mean \pm SD $34.0 \pm 9.2 \mathrm{yrs}$ ), who had engaged in indium processing in the plant for 3.6 yrs (0.8-17.2). Of these, 23 workers were nonsmokers and 85 workers were former or current smokers; the median smoking history of the latter group was 9 pack-yrs (0.3-49.5). The serum indium concentration was measured in 105 workers and all other parameters were obtained from all 108 workers. A total of 38 healthy volunteers (aged $41.7 \pm 7.9 \mathrm{yrs}$ ) were recruited from non-indium workers and administrative personnel in the same plant to obtain reference HRCT $(n=24)$ and serum indium concentrations $(n=27)$. These volunteers consisted of 10 nonsmokers and 28 former or current smokers; the smoking history of the latter was 15.5 pack-yrs (0.2-49.5). Written informed consent was obtained from all the subjects.

The working duration, respiratory symptoms and signs were examined according to the Japanese Pneumoconiosis Questionnaire [11]. Most workers had rotated among various ITO processing sections so it was difficult to analyse the relationship between specific job sites and the prevalence of disorders. However, since the reported fatal case [5] was engaged in wet-surface grinding of ITO targets, special care was taken to see whether there were any differences between workers who had mainly been engaged in surface grinding $(n=30)$ and those who had mainly been engaged in other tasks $(n=78)$.

\section{Chest radiography and HRCT of the lung}

Chest radiographs were taken to evaluate abnormal shadows in the lung fields, especially those that were reticulonodular or ground glass-like.

HRCT of the lungs was obtained at three levels: upper lung field, at the vicinity of the aortic arch; middle lung field, at the vicinity of the tracheal bifurcation; and lower lung field, $1-3 \mathrm{~cm}$ above the right hemidiaphragm. These are basically the same levels used to assess low-attenuation areas in patients with emphysema [12]. Thin-section (1-mm) slices were obtained from each bilateral lung field at a deep inspiratory level and the degrees of interstitial (ground-glass opacity or reticulonodular shadow) and emphysematous changes (lowattenuation areas) were scored visually based on the area involved: 0 , none; $1,1-10 \% ; 2,11-25 \%$; 3, 26-50\%; 4, 51-75\%; and $5,>75 \%$ of the lung field. Two pulmonologists who were naïve to the patients' histories scored the HRCT films independently and the average total score was adopted for those that differed. Spearman's rank correlations between the scores of the two examiners were 0.598 and 0.422 for interstitial and emphysematous changes, respectively $(p<0.0001)$. The difference in scores between the two examiners was 0 or 1 in $88 \%$ of subjects for the interstitial ratings and in $91 \%$ for the emphysematous ratings. The average total score that exceeded the 90th percentile of that of normal volunteers was adopted to be significant for interstitial and emphysematous changes.

\section{Pulmonary function tests}

Spirometry was performed using a respirometer (DISCOM21FX; Chest MI, Tokyo, Japan) to obtain vital capacity (VC), forced vital capacity (FVC) and forced expiratory volume in one second (FEV1). Diffusing capacity of the lung for carbon monoxide $(D \mathrm{~L}, \mathrm{CO})$ was measured by the single-breath method
(Chestac-8800; Chest MI). Functional residual capacity (FRC) was obtained by the helium dilution method and residual volume (RV) and total lung capacity (TLC) were calculated by concomitantly performed spirometry. Per cent predicted values of VC, DL,CO, FRC, RV and TLC were calculated according to predictive literature data [13-17].

\section{Measurement of serum KL-6 and serum indium}

Serum KL-6 levels were measured by an electrochemiluminescence immunoassay (Special Reference Laboratory, Tokyo, Japan). The concentration of serum indium was measured by Japan Industrial Safety and Health Association (JISHA) using inductively coupled plasma mass spectrometry (ICP-MS; Agilent Technologies, Santa Clara, CA, USA).

\section{Measurement of indium in the working environment}

In the plant presently studied, the processing of ITO includes the mixing of indium oxide (average diameter $2.0 \mu \mathrm{m}$ ) and tin oxide powder $(1.9 \mu \mathrm{m})$, pulverisation, press moulding, sintering, surface grinding and sawing; all the working rooms were equipped with an exhaust ventilation system and the workers were required to use dust respirators (DR80L2W; Shigematsu, Tokyo, Japan) compatible with the standard of Ministry of Health, Labour and Welfare (filter efficiency >95\%). Although pulverisation, surface grinding and sawing were performed in wet systems, it was recognised that ITO-containing droplets and waste water splashed around the machines could dry, resulting in ITO dust suspended in the air, particularly in the surface-grinding room. The diameter of the dust particles ranged $0.1-11 \mu \mathrm{m}$ (average $2.5 \mu \mathrm{m}$ ).

The indium in the air of eight working areas was measured by JISHA in November 2002 according to the Work Environment Measurement Law: area sampling of at least six sampling points in a work site (cross-points of 3-m mesh line), using a low volume air sampling pump (Airchek HV30; SKC Gulf Coast Inc., Houston, TX, USA) with an appropriate filter (Fiberfilm Filter T60A20; Tokyo Dylec, Tokyo, Japan), and a sampling time of $\geqslant 10 \mathrm{~min}$ was adopted at each sampling point.

\section{Statistical analysis}

For continuous data, the data distribution was examined and an appropriate transformation was performed to approximate a normal distribution before the analysis. An unpaired t-test, Welch method or one way ANOVA followed by Dunnett's test was applied to compare the differences in means. When an appropriate transformation was not valid, Wilcoxon's nonparametric test or Kruskal-Wallis rank-sum test was employed. The dose-dependent trend was assessed by applying a multiple regression model. For prevalence data, proportions were compared by Chi-squared test or Fisher's exact method. The dose-dependent trend of the proportions was examined by applying a multiple logistic regression model. Pearson's product-moment correlation coefficient or Spearman's rank correlation coefficient was used to assess the relationship between two sets of data.

\section{RESULTS}

\section{General findings}

Symptoms

In total, 18 of the indium workers, including three nonsmokers, had chronic cough and/or sputum. None of the workers 
complained of breathlessness on exertion, nor did they exhibit abnormal respiratory sounds on auscultation or peripheral cyanosis, but four had clubbed fingers.

\section{Radiological findings}

Fine reticulonodular shadows were recognised on chest radiographs in seven indium workers; four of them had chronic cough and/or sputum and one of them was a nonsmoker.

Figure 1 shows an example of a HRCT, which indicates fine reticulonodular shadows and sparse ground-glass-like areas scattered throughout the lower lung fields. Significant interstitial changes were observed in $23(21 \%)$ indium workers. All of the seven workers who had abnormalities on chest radiographs were found to have significant interstitial shadows on HRCT.

Figure 2 shows an example of the emphysematous changes on HRCT; in this case, low-attenuation areas are observed in addition to interstitial changes in the left upper lung field. There were significant emphysematous changes in $14(13 \%)$ indium workers and among them two were nonsmokers and five had chronic cough and/or sputum.

Pulmonary function tests

Six workers showed VC $<80 \%$ pred; four of the subjects showed FEV1/FVC $<70 \%$ and $D \mathrm{~L}, \mathrm{CO}<80 \%$ pred, respectively. The values of FRC and RV were both $<80 \%$ pred in only one subject and none of the subjects revealed TLC $<80 \%$ pred.

Serum KL-6 and indium levels

The serum KL-6 level was abnormally high $\left(>500 \mathrm{U} \cdot \mathrm{mL}^{-1}\right)$ in $40(37 \%)$ indium workers. The geometric mean (GM) \pm geometric SD of serum indium in the indium workers was $7.9 \pm 4.3 \mathrm{ng} \cdot \mathrm{mL}^{-1}$, which was significantly higher than the level of $0.3 \pm 2.6 \mathrm{ng} \cdot \mathrm{mL}^{-1}$ found in the healthy volunteers $(\mathrm{p}<0.001)$.
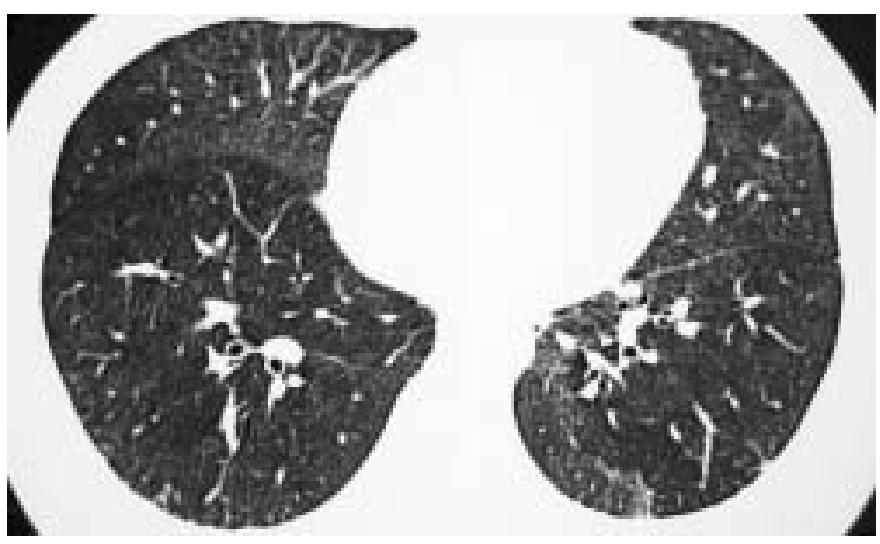

FIGURE 1. High-resolution computed tomography scans of the lower lung fields of a nonsmoking worker who had engaged in indium processing for $12.3 \mathrm{yrs}$. Results of investigations are as follows: serum indium $40 \mathrm{ng} \cdot \mathrm{mL}^{-1} ; \mathrm{KL}-6$ $1,930 \mathrm{U} \cdot \mathrm{mL}^{-1}$; vital capacity $92 \%$ predicted; residual volume $92 \%$ pred; functional residual capacity $108 \%$ pred; total lung capacity $91 \%$ pred; forced expiratory volume in one second/forced vital capacity $78 \%$; and diffusing capacity of the lung for carbon monoxide $77 \%$ pred.

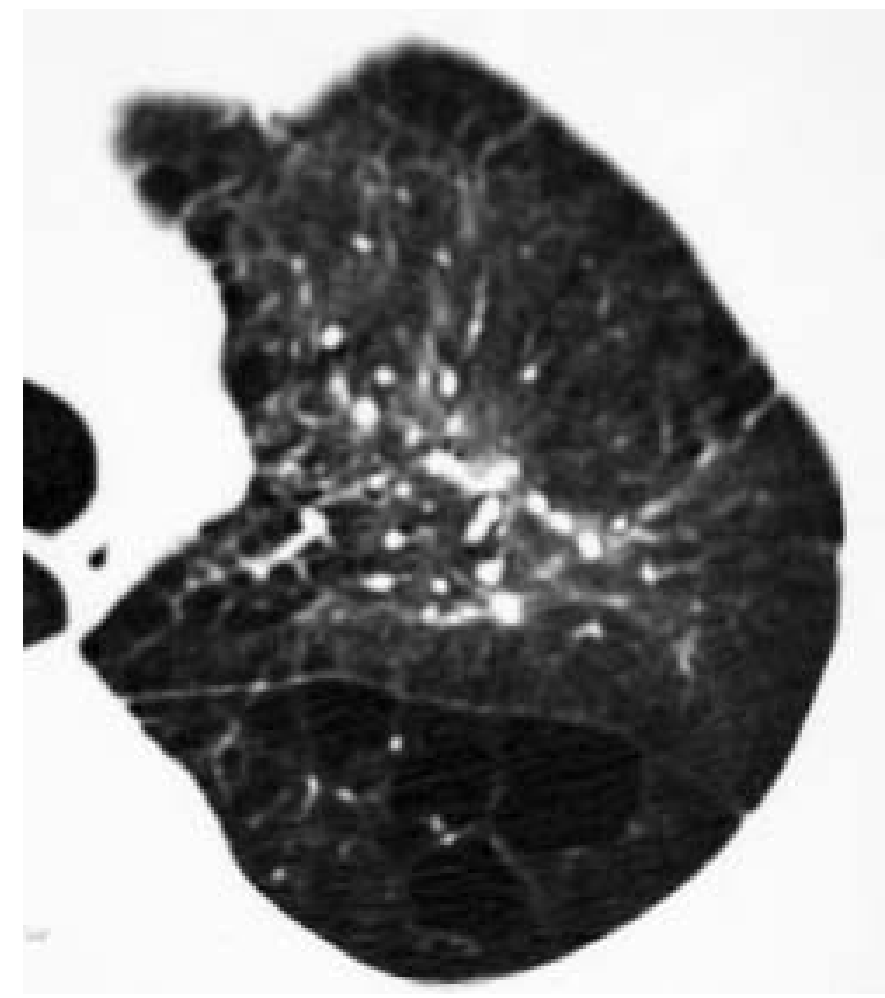

FIGURE 2. High-resolution computed tomography scans of left upper lung field of a nonsmoking worker who had engaged in indium processing for $8.3 \mathrm{yrs}$ until July 2000. Results of investigations are as follows: serum indium $99 \mathrm{ng} \cdot \mathrm{mL}^{-1}$ $\mathrm{KL}-6 \quad 1190 \mathrm{U} \cdot \mathrm{mL}^{-1}$; vital capacity 95\% predicted; residual volume $163 \%$ pred; functional residual capacity $145 \%$ pred; total lung capacity $117 \%$ pred; forced expiratory volume in one second/forced vital capacity $49 \%$; and diffusing capacity of the lung for carbon monoxide $78 \%$ pred.

\section{Analysis of working environment}

According to JISHA, the GM of indium in the eight working areas ranged $0.01-0.05 \mathrm{mg} \cdot \mathrm{m}^{-3}$. The GM indium concentrations were similar between the surface-grinding room $(0.05 \pm$ $\left.3.96 \mathrm{mg} \cdot \mathrm{m}^{-3}\right)$ and other rooms $\left(0.01 \pm 1.36-0.05 \pm 4.16 \mathrm{mg} \cdot \mathrm{m}^{-3}\right)$ and the maximum concentrations were 0.24 and $0.36 \mathrm{mg} \cdot \mathrm{m}^{-3}$, respectively. However, both interstitial and emphysematous HRCT changes were more prevalent among surface-grinding workers than other workers (interstitial changes: $40 \%$ versus $14 \%$; emphysematous changes: $27 \%$ versus $8 \%$; $<<0.01$ ). Other parameters including the serum indium concentration and KL6 were not significantly different between the two groups.

Comparison among currently indium-exposed, formerly indiumexposed and non-indium-exposed workers

The serum indium concentration was significantly higher in both formerly and currently exposed workers than in nonexposed workers, but it did not differ between former and current workers (table 1). The exposure period was also not different between current and former workers.

Similarly, neither the KL-6 level nor HRCT scores were different between current and former workers. Furthermore, there were no significant differences between former and current workers in any parameters of the pulmonary function 
tests except for TLC \% pred, which was slightly lower in former workers.

\section{Quartile analysis according to serum indium level}

The combined currently and formerly exposed indium workers were classified into four groups according to the serum indium concentration: $0.2-2.9,3.2-8.0,8.3-21.7$, and 22.2$126.8 \mathrm{ng} \cdot \mathrm{mL}^{-1}$ (table 2). The percentage of smokers was not different nor was there any difference in smoking history among the quartiles. All of the four workers with clubbed fingers belonged to the fourth quartile of the serum indium concentration.

The serum indium levels tended to increase with the duration of the exposure period; this trend was found to be statistically significant. The serum KL-6 level sharply increased in the first, second, third and fourth quartiles in this order. The scores of the interstitial and emphysematous changes on HRCT tended to increase with each higher quartile category. Per cent predicted values of TLC and DL,CO decreased with each higher quartile category, and were significantly lower in the fourth quartile as compared with the first quartile. The trends of the increases or decreases for these five parameters were all statistically significant. In addition, the prevalence of workers with KL-6 above the reference value and of those with interstitial changes on HRCT also increased with each higher quartile and the trends were statistically significant. Per cent predicted values of VC, RV and FRC and FEV1/FVC did not change significantly among the quartiles.

\section{Comparison according to HRCT changes}

A comparison was made between the indium workers with significant changes on HRCT and those without HRCT changes based on the criteria described in the Methods and materials section (table 3). The smoking index and the prevalence of smokers were not different between the two groups. In contrast, those with positive HRCT changes had experienced longer exposure to indium and had higher serum indium levels than those without HRCT changes. Similarly, those who showed HRCT changes had higher rates of KL-6 abnormalities and greater mean KL-6 values as compared with those who did not. Those with interstitial changes had a higher prevalence of significant emphysematous changes as well as greater emphysematous scores, and vice versa.

The mean \% pred values of DL,CO, TLC and RV were smaller in workers with positive interstitial changes than in those without them. The mean value of FRC \% pred was greater, that of $D \mathrm{~L}, \mathrm{CO}$ was smaller and reductions in $\mathrm{DL}, \mathrm{CO} \%$ pred as well as FEV1/FVC were more prevalent in workers with emphysematous changes as compared with those without emphysematous changes.

\section{Correlation analysis}

The correlations between the variables among the indium workers are shown in table 4. A significant correlation was found between the log serum indium concentration and the $\log$ KL-6 among the indium workers. The serum indium concentration was also correlated with the interstitial and emphysematous scores on HRCT. Diffusing capacity was inversely correlated with the degrees of both interstitial and emphysematous changes on HRCT. Per cent predicted values of TLC and RV were negatively correlated with the interstitial score on HRCT.

\section{DISCUSSION}

The present study is the first to show that interstitial pulmonary disorders characterised by HRCT findings and an elevation of KL-6 levels occurred in a large number of indium workers. Workers with higher serum indium concentrations

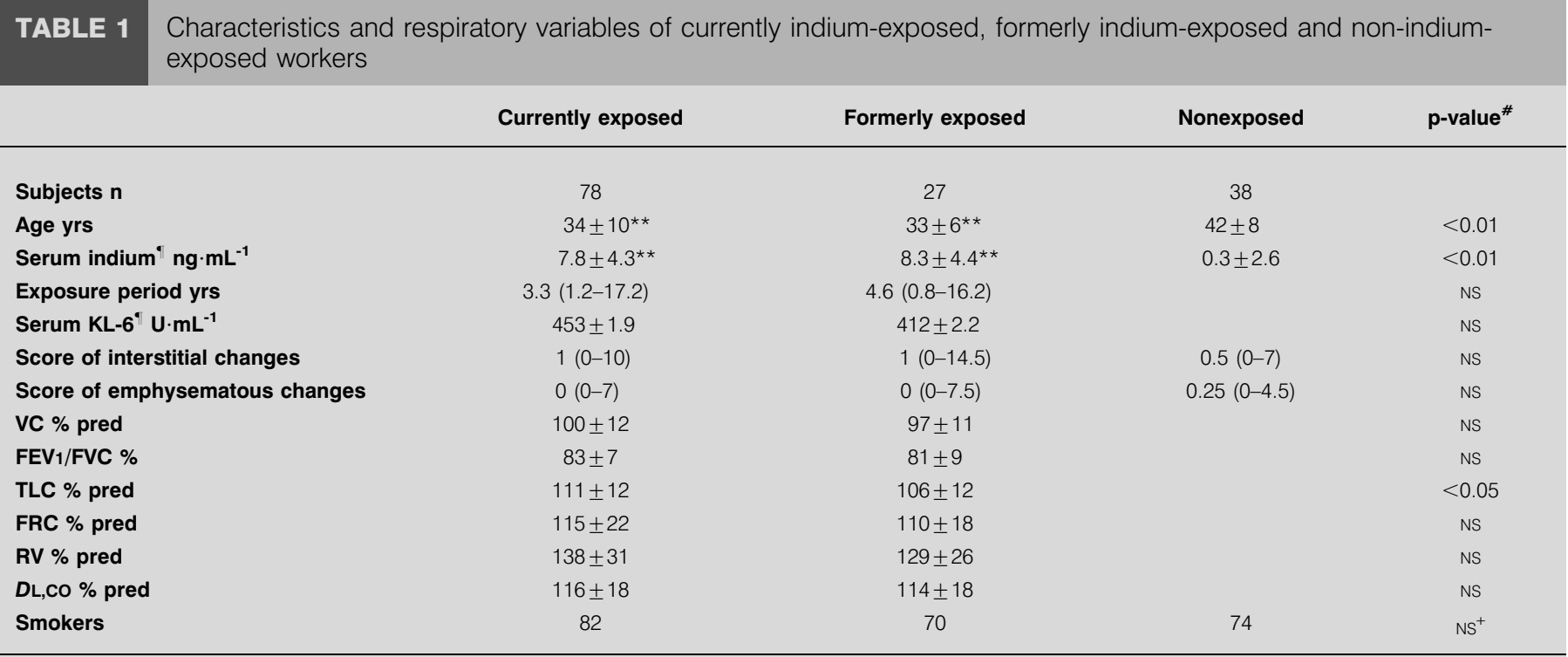

Data are presented as mean \pm SD, median (range) or \% prevalence, unless otherwise stated. VC: vital capacity; \% pred: \% predicted; FEV 1 : forced expiratory volume in one second; FVC: forced vital capacity; TLC: total lung capacity; FRC: functional residual capacity; RV: residual volume; DL,CO: diffusing capacity of the lung for carbon monoxide; NS: nonsignificant. ${ }^{\#}$ : one-way ANOVA; $\because$ : geometric mean \pm geometric SD; ${ }^{+}$: by Chi-squared test. **: p<0.01 by Dunnett's test compared with the nonexposed workers 


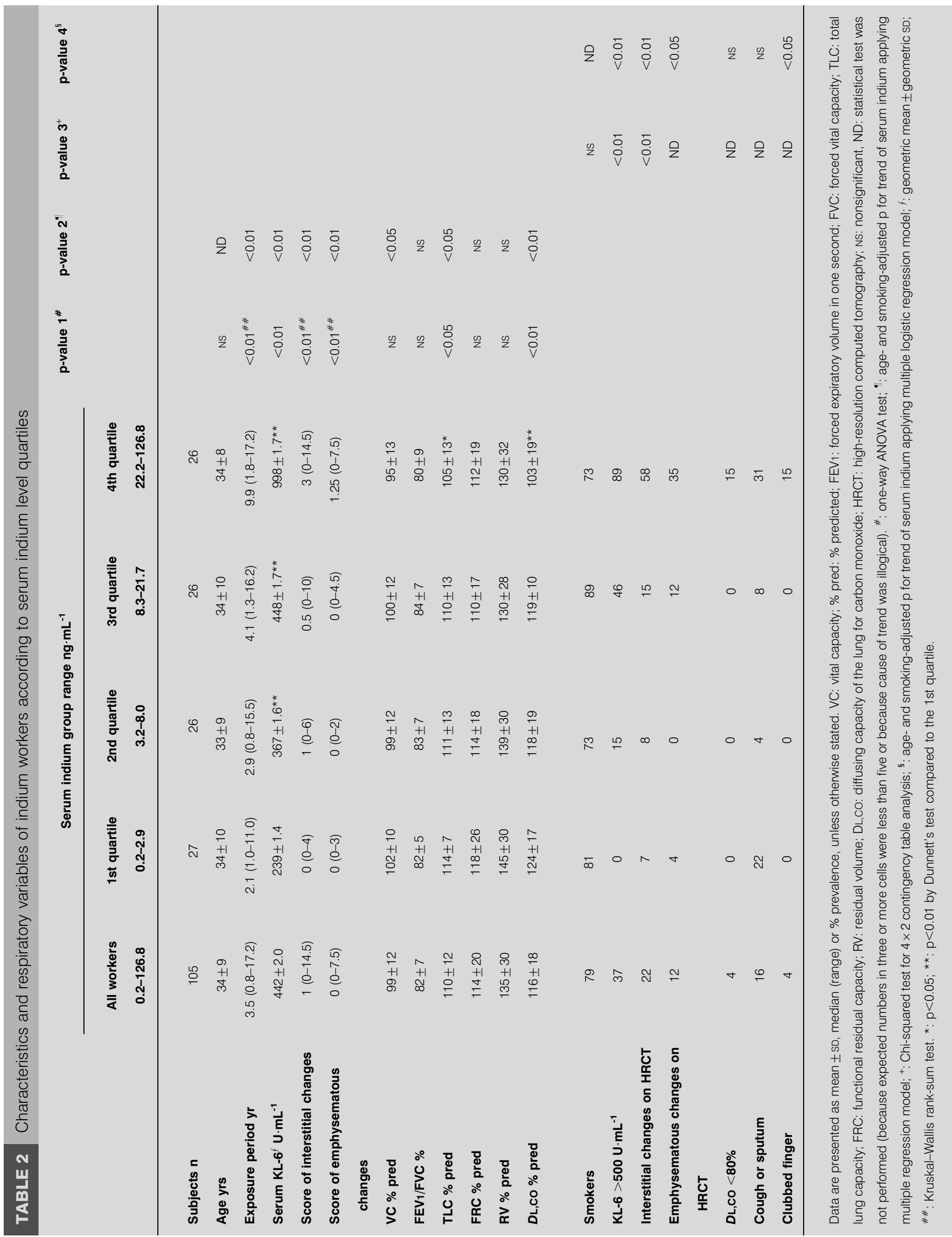


TABLE 3 Comparison according to interstitial changes or emphysematous changes on lung high-resolution computed tomography (HRCT)

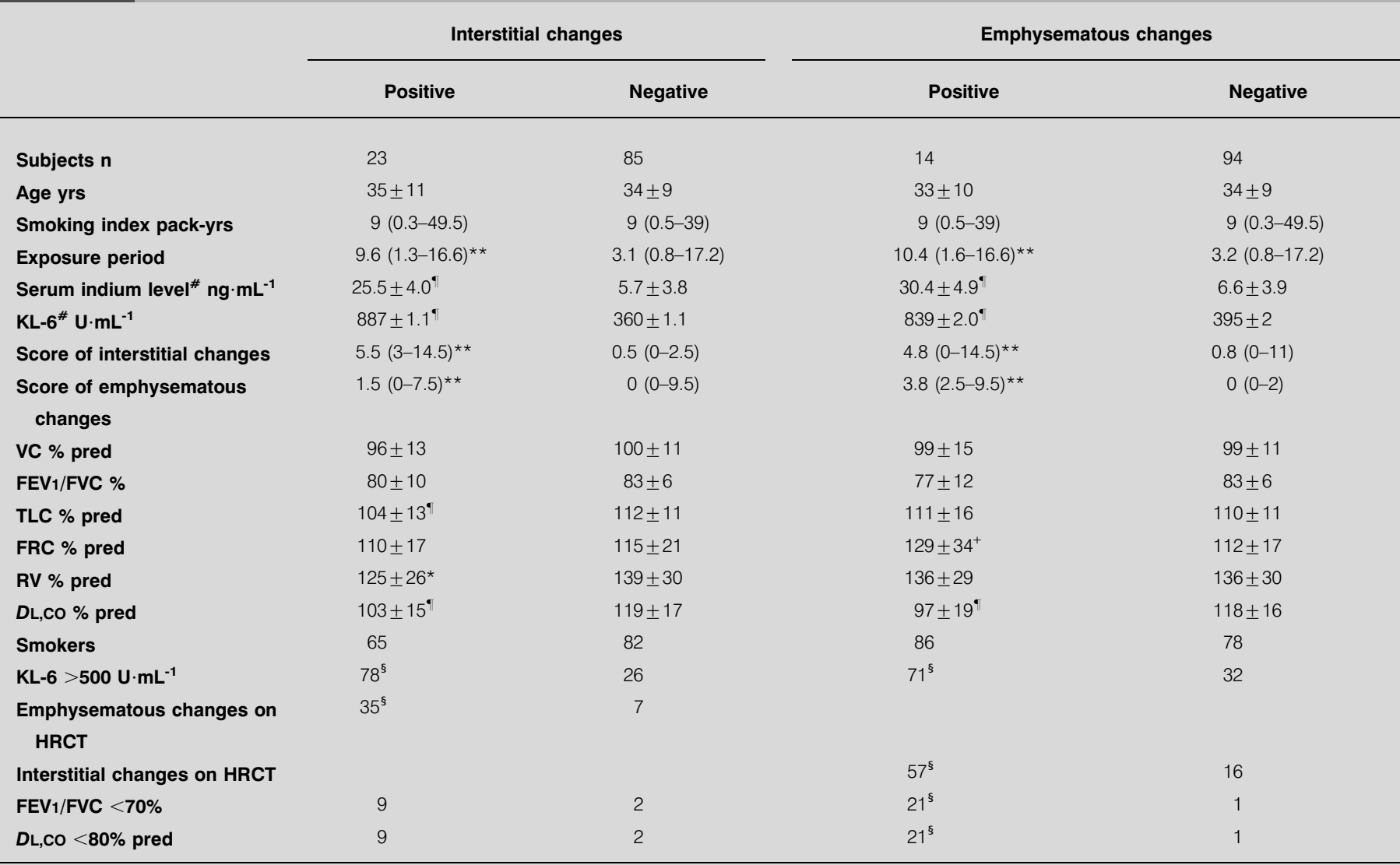

Data are presented as mean $\pm \mathrm{SD}$, median (range) or \% prevalence, unless otherwise stated. VC: vital capacity; \% pred: \% predicted; FEV1: forced expiratory volume in one second; FVC: forced vital capacity; TLC: total lung capacity; FRC: functional residual capacity; RV: residual volume; DL,CO: diffusing capacity of the lung for carbon

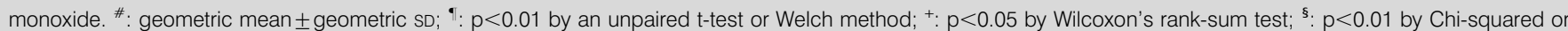
Fisher's exact method; *: $p<0.05$ by an unpaired t-test or Welch method; **: $p<0.01$ by Wilcoxon's rank-sum test.

TABLE 4 Correlations between variables

\begin{tabular}{|c|c|c|c|c|}
\hline & $r_{s}$ & p-value & $\mathbf{r}_{\mathbf{s}}$ & p-value \\
\hline Age yrs & 0.124 & NS & -0.111 & NS \\
\hline Smoking index & 0.054 & NS & 0.053 & NS \\
\hline Serum indium level & 0.427 & $<0.0001$ & 0.386 & $<0.0001$ \\
\hline TLC \% pred & -0.299 & $<0.01$ & 0.054 & NS \\
\hline FRC \% pred & -0.138 & NS & 0.231 & 0.016 \\
\hline RV \% pred & -0.255 & 0.008 & 0.13 & NS \\
\hline
\end{tabular}

HRCT: high-resolution computed tomography; $D L, C O$ : diffusing capacity of the lung for carbon monoxide; \% pred: \% predicted; TLC: total lung capacity; FRC: functional residual capacity; RV: residual volume; Ns: nonsignificant. Interstitial score versus emphysematous score: $r_{s}=0.342 ; p<0.001 . \log$ (serum indium level) versus log(KL-6): $r=0.703 ; p<0.0001$. 
had significantly longer exposure periods, greater HRCT changes, lower DL,CO and higher KL-6 levels compared with those with lower serum indium concentrations. Moreover, the degree of HRCT change and the level of serum KL-6 were significantly correlated with the serum indium level.

KL-6 is mainly produced by alveolar type II cells and its level increases in the serum in the presence of lung injury through the rise in alveolar-capillary permeability and the production is augmented by regenerated alveolar type II cells $[8,9]$. It appeared that ITO particles deposited in the alveoli evoked persistent lung injury and an increase in the serum KL-6 levels in many indium workers in the present study.

KAZEROONI et al. [18] have reported that a limited thin-section computed tomography scoring system like the one adopted in the present study correlates well with the pathological changes of idiopathic pulmonary fibrosis. A similar scoring method has been reported to assess the low-attenuation areas in patients with pulmonary emphysema [12]. Furthermore, these scores appeared to express the extent of the disease in so far as the degree of HRCT changes assessed with the method used had a dose-response relationship with the serum indium level and it was correlated with other indices of interstitial lung disease such as the serum KL-6 level and DL,CO.

The functional impairments were mild in subjects of the present study; TLC and DL,CO showed a dose-dependent reduction with the serum indium level but the mean \% pred values of TLC and DL,CO were within normal ranges, even in the highest quartile of the serum indium level. Although the predicted values may be inappropriate for $D \mathrm{~L}, \mathrm{CO}, \mathrm{RV}, \mathrm{FRC}$ and TLC because these reference values were not obtained from the Japanese population, the mixed disorder, comprising interstitial and emphysematous lesions, may in part have minimised the lung volume changes.

Some of the affected workers were nonsmokers and neither the interstitial nor the emphysematous scores of the lung HRCT were correlated with the smoking histories, suggesting that the pulmonary disorders found in the present study were only marginally, if at all, related to the adverse effects of smoking. Conversely, the emphysematous score was correlated with the interstitial score of the lung HRCT as well as with the serum indium concentration (table 4). The emphysematous changes may be evoked by bronchiolar obstruction due to interstitial and intra-alveolar cholesterol granulomas, as has been described previously $[5,6]$.

Although the HRCT changes were more prevalent in the surface-grinding workers than in other workers, this finding was not found to be related to the indium concentration in the air. The measured indium concentrations in the work sites in the present study would not necessarily correspond to the indium burden in the lungs because the biological half-life of indium in the lungs is long [4]. It is likely that there was more environmental indium in the past, considering the recent efforts that have been made to improve the working conditions including semi-closure of open systems such as the wetsurface-grinding process and strict implementation of dust respirator usage. These improvements, together with the rotation of most workers among various sections of the ITO processing system, may have obscured the relationships between the environmental indium concentration and the serum indium concentration or the extent of pulmonary disorders. Therefore, no definitive conclusion can be drawn concerning the relationship between the workplace indium concentration and the respiratory effects from the results of the present study.

The contribution of tin oxide to the pathogenesis of interstitial pulmonary disease in the workers studied cannot be completely ruled out. However, it has been reported that stannosis is associated with little fibrosis $[19,20]$, thereby differing from the pulmonary interstitial changes and fibrosis observed in the present study and other reported cases $[5,6]$. Thus, it is likely that the pulmonary disorders observed in the present study were evoked mainly by indium oxide rather than by tin oxide.

ZHENG et al. [21] have reported the distribution of indium following oral and intratracheal administration of indium phosphide in rats and described that indium is poorly absorbed from the gastro-intestinal tract and that indium ions are uniformly distributed among tissues once entering the circulation. It appears that the route of administration of indium was almost exclusively through the airway in subjects of the present study. In fact, there was a substantial concentration of indium particles in the working environment even at the time of the study. It has been estimated that the elimination of deposited indium from the lung is slow, and the clearance half-life is $>200$ days in rats [4]. In the present study, there were no significant differences in the serum indium concentration or the exposure period between currently exposed and formerly exposed workers, suggesting the slow clearance of deposited indium in the body. It is also suggested that the serum indium level reflects chronic rather than acute exposure in so far as the serum indium concentration was closely related to the working duration and did not depend on whether the exposure occurred in the past or was still ongoing.

Pulmonary toxicity of various indium compounds including indium trichloride $[1,2]$, indium phosphide [3, 4], ITO [3] and indium arsenide [22], either inhaled or intratracheally administered, has been reported in animal experiments. However, there are little data showing whether ITO or insoluble indium metal differs from other indium compounds in terms of biological effects. The present study is the first to explore the possible pulmonary effects of indium compounds in a significant number of humans.

Homma et al. [5] reported a case of severe interstitial pneumonia that occurred in an indium worker who died of bilateral pneumothorax. The pneumothorax might have been evoked by emphysematous changes of the lung such as those described in the present report. The serum indium concentration in the case of Homma et al. [5] was $290 \mathrm{ng} \cdot \mathrm{mL}^{-1}$, which is significantly higher than that of subjects of the present study; therefore, the modest pulmonary impairments found in the latter subjects may be explained by the lower degree of indium burden. However, it is noteworthy that a significant number of former workers still had elevations of KL-6 as well as changes on HRCT, suggesting the chronic persistence of these changes. Moreover, the pathological findings of some of the current authors' subjects reported elsewhere [23] were similar to those of previously reported cases [5,6], namely the accumulation of 
cholesterol-laden macrophages in the alveoli with interstitial fibrosis and cholesterol granulomas in transbronchially biopsied and/or surgically resected lung specimens. The lung interstitial as well as emphysematous changes may worsen and symptoms may become more evident in the future, considering the relatively short duration of exposure (median $3.6 \mathrm{yrs}$ ) and the early examination (18 yrs since the beginning of ITO processing in this plant). Therefore, careful follow-up, together with an effort to eliminate exposure to aerosolised indium, is needed.

In conclusion, the results of the present study show that interstitial pulmonary disorders occurred in a significant number of indium-processing workers, and that inhaled indium could be a potential cause of occupational lung disease.

\section{ACKNOWLEDGEMENTS}

The authors wish to express their great appreciation to T. Takata, H. Sakurai and K. Honma for their valuable comments, A. Kanahara for his clinical contribution, M. Kuramochi for radiological assessment and to B. Bell for reading the manuscript.

\section{REFERENCES}

1 Blazka ME, Dixon D, Haskins E, Rosenthal GJ. Pulmonary toxicity to intratracheally administered indium trichloride in Fischer 344 rats. Fundam Appl Toxicol 1994; 22: 231-239.

2 Blazka ME, Tepper JS, Dixon D, Winsett DW, O'Connor RW, Luster MI. Pulmonary response of Fischer 344 rats to acute nose-only inhalation of indium trichloride. Environ Res 1994; 67: 68-83.

3 Tanaka A, Hirata M, Omura M, et al. Pulmonary toxicity of indium-tin oxide and indium phosphide after intratracheal instillations into the lung of hamsters. J Occup Health 2002; 44: 99-102.

4 National Toxicology Program. Toxicology and carcinogenesis studies of indium phosphide (CAS No. 22398-80-7) in $\mathrm{F} 344 / \mathrm{N}$ rats and $\mathrm{B}_{6} \mathrm{C}_{3} \mathrm{~F}_{1}$ mice (inhalation studies). $\mathrm{NIH}$ Publication No. 01-4433. U.S. Department of Health and Human Services, Public Health Service, National Institute of Health, Bethesda, MD, 2001. http://ntp-server.niehs. nih.gov. Date last accessed: July 2006; date last updated: October 2004.

5 Homma T, Ueno T, Sekizawa K, Tanaka A, Hirata M. Interstitial pneumonia developed in a worker dealing with particles containing indium-tin oxide. J Occup Health 2003; 45: 137-139.

6 Homma S, Miyamoto A, Sakamoto S, Kishi K, Motoi N, Yoshimura K. Pulmonary fibrosis in an individual occupationally exposed to inhaled indium-tin oxide. Eur Respir J 2005; 25: 200-204.

7 Kohno N, Kyoizumi S, Awaya Y, Fukuhara H, Yamakido M, Akiyama M. New serum indicator of interstitial pneumonitis activity. Sialylated carbohydrate antigen KL-6. Chest 1989; 96: 68-73.

8 Ohnishi H, Yokoyama A, Kondo $\mathrm{K}$, et al. Comparative study of KL-6, surfactant protein-A, surfactant protein D, and monocyte chemoattractant protein-1 as serum markers for interstitial lung diseases. Am J Respir Crit Care Med 2002; 165: 378-381.

9 Hermans C, Bernard A. Lung epithelium-specific proteins: characteristics and potential applications as markers. Am J Respir Crit Care Med 1999; 159: 646-678.

10 Chonan $\mathrm{T}$, Taguchi O. Interstitial pneumonia occurred in indium tin oxide workers. J Jap Resp Soc 2004; 42: Suppl. P147, 185.

11 The Labor Hygiene Section in the Safety Hygiene Department of the Ministry of Labour. A Handbook for Examining Pneumoconiosis. Tokyo, Japan Industrial Safety and Health Association, 1980.

12 Goddard PR, Nicholson EM, Laszlo G, Watt I. Computed tomography in pulmonary emphysema. Clin Radiol 1982; 33: 379-387.

13 The Japanese Respiratory Society. Guidelines for respiratory function tests-spirometry, flow volume curve, diffusing capacity of the lung. Tokyo, Medical Review Co. Ltd, 2004.

14 Burrows B, Kasik JE, Niden AH, Barclay WR. Clinical usefulness of the single-breath pulmonary diffusing capacity test. Am Rev Respir Dis 1961; 84: 789-806.

15 Grimby G, Soderholm B. Spirometric studies in normal subjects. III. Static lung volumes and maximum voluntary ventilation in adults with a note on physical fitness. Acta Med Scand 1963; 173: 199-206.

16 Goldman HI, Becklake MR. Respiratory function tests: normal values at median altitudes and the prediction of normal results. Am Rev Tuberc 1959; 79: 457-467.

17 Rossier PH, Buhlmann AA, Wiesinger K. Spirometry. In: Luchsinger PC, Moser KM, eds. Respiration: Physiologic Principles and their Clinical Applications. 2nd Edn. St Louis, Mosby, 1960; pp. 127-150.

18 Kazerooni EA, Martinez FJ, Flint A, et al. Thin-section CT obtained at $10-\mathrm{mm}$ increments versus limited three-level thin-section CT for idiopathic pulmonary fibrosis: correlation with pathologic scoring. ARJ Am J Roentgenol 1997; 169: 977-983.

19 Robertson AJ, Rivers D, Nagelschmidt G, Duncumb P. Stannosis: benign pneumoconiosis due to tin dioxide. Lancet 1961; 1: 1089-1093.

20 Howe P, Watts P. Evaluation of health effects. In: Tin and Inorganic Compounds (Concise International Chemical Assessment Document; 65). Geneva, World Health Organization, 2005; pp. 33-37.

21 Zheng W, Winter SM, Kattnig MJ, Carter DE, Sipes IG. Tissue distribution and elimination of indium in male Fischer 344 rats following oral and intratracheal administration of indium phosphide. J Toxicol Environ Health 1994; 43: 483-494.

22 Yamazaki K, Tanaka A, Hirata M, et al. Long term pulmonary toxicity of indium arsenide and indium phosphide instilled intratracheally in hamsters. J Occup Health 2000; 42: 169-178.

23 Taguchi O, Chonan T. Three cases of indium lung. J Jap Respir Soc 2006; 44: 532-535. 